\title{
COMPLETE CONFORMAL METRICS WITH ZERO SCALAR CURVATURE
}

\author{
XIAOYUN MA AND ROBERT C. MCOWEN
}

(Communicated by Clifford J. Earle, Jr.)

\begin{abstract}
In this paper we consider the problem of when a noncompact Riemannian manifold $\widehat{M}$ admits a complete conformal metric with zero scalar curvature. In particular, we show that this can be achieved if $\widehat{M}$ is the noncompact manifold obtained by deleting a smooth submanifold $\Gamma^{n}$ from a compact Riemannian manifold $M^{N}$ provided $n \leq(N-2) / 2$ and the Sobolev quotient is positive.
\end{abstract}

\section{INTRODUCTION}

Suppose $(M, g)$ is a compact Riemannian manifold of dimension $N \geq 3$ and suppose $\Gamma$ is a closed smooth submanifold of dimension $n$. Then $\widehat{M}=$ $M \backslash \Gamma$ is a noncompact manifold, and we want to consider Riemannian metrics $\hat{g}$ on $\widehat{M}$. Let $S$ denote the scalar curvature of $g$ multiplied by the constant $(N-2) / 4(N-1)$ and $\hat{S}$ the corresponding quantity for $\hat{g}$. We consider the following basic

Problem. Find a complete metric $\hat{g}$ that is conformal to $g$ on $\widehat{M}$ and has constant $\widehat{S}$.

This problem was first considered by Loewner and Nirenberg [LN] when $M$ is the sphere $S^{N}$ and $\widehat{S} \equiv-1$ and then generalized by Aviles and McOwen [AM] to arbitrary $M$. The result is the following: $\widehat{M}$ admits a complete conformal metric $\hat{g}$ with $\widehat{S} \equiv-1$ if and only if $n>(N-2) / 2$.

In attempting to study the case $\widehat{S} \geq 0$, one immediately encounters a constraint involving the "conformal Laplacian"

$$
L_{g}=-\Delta_{g}+S
$$

(notice we have absorbed the dimensional constant $(N-2) / 4(N-1)$ into our definition of $S$ ). Let us consider the 1st Dirichlet eigenvalue

$$
\lambda_{0}=\lambda_{0}(\widehat{M}, g)=\inf _{\varphi \in C_{0}^{\infty}(\widehat{M})} \frac{\int_{\widehat{M}}\left(|\nabla \varphi|^{2}+S \varphi^{2}\right) d V}{\int_{\widehat{M}} \varphi^{2} d V}
$$

Received by the editors May 17, 1990.

1991 Mathematics Subject Classification. Primary 35J99, 58G99.

Key words and phrases. Conformal metrics, conformal Laplacian, scalar curvature, Riemannian manifolds. 
and the Sobolev quotient

$$
Q_{0}=Q_{0}(\widehat{M}, g)=\inf _{\varphi \in C_{0}^{\infty}(\widehat{M})} \frac{\int_{\widehat{M}}\left(|\nabla \varphi|^{2}+S \varphi^{2}\right) d V}{\left(\int_{\widehat{M}} \varphi^{2 N /(N-2)} d V\right)^{(N-2) / N}},
$$

where of course $|\nabla *|$ and $d V$ are in terms of $g$. The Sobolev quotient is a conformal invariant, i.e., $Q_{0}$ depends only on the conformal class of $g$ on $\widehat{M}$, so we shall write $Q_{0}=Q_{0}(\widehat{M}) ; \lambda_{0}$ is not a conformal invariant, but the condition $\lambda_{0}<0$ is conformally invariant and equivalent to $Q_{0}<0$. The constraint for $\widehat{S} \geq 0$ may be expressed as follows:

Proposition. If $(\widehat{M}, g)$ admits a conformal metric $\hat{g}$ with $\widehat{S} \geq 0$ then $Q_{0}(\widehat{M}) \geq$ 0 .

To prove the proposition, let us recall that if $\hat{g}=u^{4 /(N-2)} g$ where $u$ is a positive function, then

$$
\Delta_{g} u+\widehat{S} u^{(N+2) /(N-2)}=S u .
$$

Now if $Q_{0}<0$ then $\lambda_{0}<0$, so there is a smooth submanifold with boundary $M_{0} \subset \widehat{M}$ such that the $\lambda_{0}\left(M_{0}, g\right)<0$. Let $v$ be the eigenfunction corresponding to $\lambda_{0}\left(M_{0}, g\right): v>0$ in $M_{0}, v=0$ on $\partial M_{0}$, and $\left(-\Delta_{g}+S\right) v=\lambda_{0} v$. Using Green's Identity (with $\nu=$ exterior normal and $d \sigma=$ induced measure on $\left.\partial M_{0}\right)$

$$
\begin{aligned}
0 & >\int_{\partial M_{0}}\left(u \frac{\partial v}{\partial \nu}-v \frac{\partial u}{\partial \nu}\right) d \sigma=\int_{M_{0}}\left(u \Delta_{g} v-v \Delta_{g} u\right) d V \\
& =\int_{M_{0}}\left(-\lambda_{0} u v+\widehat{S} v u^{(N+2) /(N-2)}\right) d V
\end{aligned}
$$

so we cannot have $\widehat{S} \geq 0$ in $M_{0}$.

Notice that $Q_{0}$ is defined for the noncompact manifold $\widehat{M}$. However, using cutoff functions we can show that if $n<N-2$ then $Q_{0}(\widehat{M})=Q(M) \equiv$ $\inf _{\varphi \in C^{\infty}(M)} Q(\varphi)$ where

$$
Q(\varphi)=\frac{\int_{M}\left(|\nabla \varphi|^{2}+S \varphi^{2}\right) d V}{\left(\int_{M} \varphi^{2 N /(N-2)} d V\right)^{(N-2) / N}} .
$$

(In fact, if $r(x)$ is a smooth positive function on $\widehat{M}$ that equals the geodesic distance to $\Gamma$ in a neighborhood of $\Gamma$, then we can find $\psi_{j} \in C_{0}^{\infty}(M)$ satisfying $\left|\nabla \psi_{j}\right| \leq C j, \psi_{j}(x)=1$ for $r(x)>2 / j$ and $\psi_{j}(x)=0$ for $r(x)<1 / j$, so that

$$
\int_{M}\left|\nabla \psi_{j}\right|^{2} d V \leq C j^{2} \int_{1 / j}^{2 / j} r^{N-n-1} d r \leq C_{1} j^{n-N+2} .
$$

Since $Q_{0}(\widehat{M}) \geq Q(M)$ and if $n<N-2$ we have $Q\left(\psi_{j} \varphi\right) \rightarrow Q(\varphi)$, then $Q_{0}(\widehat{M})=Q(M)$.) Hence we could replace $Q_{0}(\widehat{M})$ by $Q(M)$ in the theorem below.

The case $Q_{0}=0$ appears to be quite subtle, as exemplified by the following (cf. [J]): If $\Gamma$ is a point in the torus $T^{N}$, then $T^{N} \backslash \Gamma$ does not admit a complete conformal metric with constant scalar curvature. However, if $Q_{0}>0$ then Lee and Parker [LP] and Jin [J] have obtained the following: If $\Gamma$ is a finite number 
of points in $M$ and $Q_{0}>0$ then there is a complete conformal metric $\hat{g}$ on $\widehat{M}$ with $\widehat{S} \equiv 0$.

The purpose of this paper is to generalize this last result and complement the result of $[\mathrm{AM}]$ by letting $\Gamma$ be a closed smooth submanifold of dimension $n \leq(N-2) / 2$ as follows:

Theorem. If $\Gamma$ is a closed smooth $n$-dimensional submanifold of the compact Riemannian manifold $(M, g)$ of dimension $N$, then $\widehat{M}=M \backslash \Gamma$ admits a complete conformal metric $\hat{g}$ with constant zero scalar curvature provided $n \leq$ $(N-2) / 2$ and $Q(M)>0$.

The proof of this theorem requires an analysis near $\Gamma$. If we use $r(x)$ as above, then the conformal metric $\hat{g}=r^{-2} g$ is complete. In [MM] the manifold $(\widehat{M}, \hat{g})$ was described as having a "warped cylindrical end" since $\hat{g}$ is asymptotic to $e^{2 z} d \theta^{2}+d z^{2}+d \omega^{2}$ (where $r=e^{-z}, d \theta^{2}$ is the induced metric on $\Gamma$, and $d \omega^{2}$ is the standard metric on the sphere $S^{N-n-1}$ ) and the conformal Laplacian $L_{\hat{g}}$ was studied using weighted Sobolev spaces: these results are summarized and somewhat generalized in $\S 1$ of this paper. In $\S 2$ we discuss a Sobolev inequality and regularity result for those weighted spaces, and in $\S 3$ we complete the proof of the theorem.

Remark 1 . Notice that $\Gamma$ may be disconnected, in which case $\widehat{M}$ has multiple ends.

Remark 2. After completing this work, we received a preprint from Ph. Delanoë entitled Generalized stereographic projections with prescribed scalar curvature, which will appear in Geometry, Physics, and Nonlinear PDE's, edited by V. Oliker and A. Treibergs, a volume in the Contemporary Mathematics series published by the American Mathematical Society. In this work, Delanoë obtains a more direct proof of the above theorem by appealing to some calculations of R. Schoen and S. T. Yau (in Manuscripta Math. 28 (1979), 159-183) and then goes on to generalize the result to achieve prescribed scalar curvature functions that vanish sufficiently quickly along $\Gamma$.

\section{MANIFOLDS WITH WARPED CYLINDRICAL ENDS}

Let us write $\widehat{M}=M_{0} \cup U$ where $M_{0}$ is a compact submanifold with boundary and $U=\{x \in \widehat{M}: 0<r(x)<\varepsilon\}$. Provided $\varepsilon$ is small enough, we may identify $U$ with the tubular neighborhood $\left\{(\theta, \xi): \theta \in \Gamma, \xi \in N_{\theta}(\Gamma), 0<|\xi|<\varepsilon\right\}$ where $N_{\theta}(\Gamma)$ is the normal space to $\Gamma$ at $\theta$ and $|\xi|^{2}=g_{i j}(\theta) \xi^{i} \xi^{j}$. In this way we may write $U=\Gamma \times(0, \varepsilon) \times \Omega$ where $\Omega$ is the sphere $S^{N-n-1}$. If we let $d \theta^{2}$ denote the metric on $\Gamma$ induced by $g$ on $M$ and $d \omega^{2}$ denote the standard metric on $S^{N-n-1}$, then $\hat{g}$ is asymptotic to

$$
\hat{g}_{0}=e^{2 z} d \theta^{2}+d z^{2}+d \omega^{2}
$$

(where $r=e^{-z}$ ), whence the term "warped cylindrical end."

Let $\eta_{0}, \eta_{1}$ be a partition of unity on $\widehat{M}$ with $\eta_{0} \in C_{0}^{\infty}(\widehat{M})$ and $\eta_{1}=0$ on $M_{0}$, and take the closure of $C_{0}^{\infty}(\widehat{M})$ in

$$
\|u\|_{\delta}^{2}=\int_{\widehat{M}}\left|\eta_{0} u\right|^{2} d \widehat{V}+\int_{U} r^{-2 \delta}\left|\eta_{1} u\right|^{2} d \widehat{V}
$$


(where $d \widehat{V}$ is the Riemannian measure of $\hat{g}$ ) to define $L_{\delta}^{2}(\widehat{M})$ for $\delta \in \mathbb{R}$. Similarly, for positive integers $s$, define $W_{s, \delta}^{2}(\widehat{M})$ as the closure of $C_{0}^{\infty}(\widehat{M})$ in the norm

$$
\|u\|_{s, \delta}^{2}=\sum_{l=0}^{s}\left\|\left|\nabla^{l} u\right|_{\hat{g}}\right\|_{\delta}^{2}
$$

where $\nabla$ is the covariant derivative for $\hat{g}$ and $\left|\nabla^{l} u\right|_{\hat{g}}^{2}=\nabla^{i_{1}} \ldots \nabla^{i_{l}} u \nabla_{i_{1}} \cdots \nabla_{i_{l}} u$.

Now, since $\hat{g}=r^{-2} g=u^{4 /(N-2)} g$ where $u=r^{-(N-2) / 2}$, we can compute $\widehat{S}$ using $(0.2)$

$$
\begin{aligned}
\widehat{S} & =u^{-(N+2) /(N-2)}\left(S u-\Delta_{g} u\right) \\
& =S r^{2}-r^{(N+2) / 2}\left(\frac{(N-2) N}{4} r^{-(N+2) / 2}-\frac{N-2}{2} r^{-(N+2) / 2} r \Delta_{g} r\right) \\
& =S r^{2}-\frac{N-2}{2}\left(\frac{N}{2}-r \Delta r\right) \rightarrow \frac{N-2}{2}\left(\frac{N}{2}-n-1\right)
\end{aligned}
$$

where we have used $r \Delta r \rightarrow N-n-1$ as $r \rightarrow 0$ (which is proved for the Euclidean case in [LN] but generalizes easily). If we let $\widehat{S}_{0}=\frac{N-2}{2}\left(\frac{N}{2}-n-1\right)$, then the condition $n \leq(N-2) / 2$ is equivalent to $\widehat{S}_{0} \geq 0$, which guarantees that the quantity

$$
\nu_{0}=\sqrt{n^{2}+4 \widehat{S}_{0}} / 2=\frac{N-n-2}{2}
$$

is positive. Hence the results of [MM] show that the conformal Laplacian defines an operator

$$
L_{\hat{g}}: W_{2, \delta}^{2}(\widehat{M}) \rightarrow L_{\delta}^{2}(\widehat{M}),
$$

which is Fredholm of index zero provided $-\nu_{0}<\delta<\nu_{0}$.

The first result of this paper is to generalize (1.4) to the operator

$$
L_{\hat{g}}: W_{s+2, \delta}^{2}(\widehat{M}) \rightarrow W_{s, \delta}^{2}(\widehat{M}) .
$$

Theorem 1.1. The operator (1.5) is Fredholm of index zero for every nonnegative integer $s$ provided $n \leq(N-2) / 2$ and $-\nu_{0}<\delta<\nu_{0}$.

Proof. In [MM] the mapping (1.4) is studied by assuming $g$ equals (1.1) in $U$ (the more general case being achieved by perturbation theory) and then constructing a Fredholm inverse $T$ by patching together $Q$, an exact inverse of $L=L_{\hat{g}}$ in $U$, with $P$, a parametrix for $L_{g}$ on $M_{0}$. The required boundedness is achieved by establishing the a priori inequality (cf. [MM, (4.5)])

$$
\|u\|_{2, \delta} \leq C\left(\|u\|_{\delta}+\|L u\|_{\delta}\right)
$$

for all $u$ vanishing outside $U$ such that $u \in L_{\delta}^{2}$ and $L u \in L_{\delta}^{2}$. Similarly, to prove Theorem 1.1 we need to prove

$$
\|u\|_{s+2, \delta} \leq C\left(\|u\|_{\delta}+\|L u\|_{s, \delta}\right)
$$

for all $u$ vanishing outside $U$ such that $u \in L_{\delta}^{2}$ and $L u \in W_{s, \delta}^{2}$.

We prove (1.7) by induction on $s$. The case $s=0$ is just (1.6), whereas for $s=1$ we must show

$$
\left\|D^{3} u\right\|_{\delta} \leq C\left(\|u\|_{\delta}+\|L u\|_{1, \delta}\right)
$$


where $D^{3}$ is any product of $r \partial_{\theta}, r \partial_{r}$, and $\partial_{\omega}$ (we have replaced covariant derivatives by partial derivatives as they differ by lower-order terms that may be controlled by the $s=0$ case). But $L u \in W_{1, \delta}^{2}$ implies $L(D u) \in L_{\delta}^{2}$ (since $[L, D]$ is second-order and we know $u \in W_{2, \delta}^{2}$ ) so we may apply (1.6) to $D u$

$$
\left\|D^{3} u\right\|_{\delta}=\left\|D^{2}(D u)\right\|_{\delta} \leq C\left(\|D u\|_{\delta}+\|L(D u)\|_{\delta}\right) .
$$

Moreover, $\|D u\|_{\delta} \leq C\left(\|u\|_{\delta}+\|L u\|_{\delta}\right)$ and

$$
\begin{aligned}
\|L(D u)\|_{\delta} & \leq\|[L, D] u\|_{\delta}+\|D(L u)\|_{\delta} \\
& \leq C\left(\|u\|_{2, \delta}+\|L u\|_{1, \delta}\right) \leq C\left(\|u\|_{\delta}+\|L u\|_{1, \delta}\right),
\end{aligned}
$$

so we obtain (1.8). Next, for $s \geq 2$ we must show that $\|u\|_{s, \delta} \leq$ $C\left(\|u\|_{\delta}+\|L u\|_{s-2, \delta}\right)$ and $\|u\|_{s+1, \delta} \leq C\left(\|u\|_{\delta}+\|L u\|_{s-1, \delta}\right)$ imply

$$
\left\|D^{s+2} u\right\|_{\delta} \leq C\left(\|u\|_{\delta}+\|L u\|_{s, \delta}\right) \text {. }
$$

But $L u \in W_{s, \delta}^{2} \subset L_{\delta}^{2}$ implies $u \in W_{2, \delta}^{2}$, so we may apply (1.7), with $s-2$ in place of $s$, to $D^{2} u$,

$$
\left\|D^{s+2} u\right\|_{\delta} \leq\left\|D^{2} u\right\|_{s, \delta} \leq C\left(\left\|D^{2} u\right\|_{\delta}+\left\|L D^{2} u\right\|_{s-2, \delta}\right) .
$$

Moreover, $\left\|D^{2} u\right\|_{\delta} \leq C\left(\|u\|_{\delta}+\|L u\|_{\delta}\right)$ and

$$
\begin{aligned}
\left\|L D^{2} u\right\|_{s-2, \delta} & \leq\left\|\left[L, D^{2}\right] u\right\|_{s-2, \delta}+\left\|D^{2} L u\right\|_{s-2, \delta} \\
& \leq C\left(\|u\|_{s+1, \delta}+\|L u\|_{s, \delta}\right) \leq C\left(\|u\|_{\delta}+\|L u\|_{s, \delta}\right),
\end{aligned}
$$

so we obtain (1.9). Thus we obtain (1.7) by induction and hence (1.5) is Fredholm.

The proof that (1.5) has zero Fredholm index is exactly the same as the proof of Theorem 5.2 in [MM], so this completes the proof of Theorem 1.1.

We, in fact, would like to know that (1.5) is an isomorphism. Suppose $u \in$ $W_{2,0}^{2}(\widehat{M})$ and $L_{\hat{g}} u=0$. Let $u_{j} \in C_{0}^{\infty}(\widehat{M})$ with $u_{j} \rightarrow u$ in $W_{2,0}^{2}(\widehat{M})$. Then

$$
\begin{aligned}
0 & =\int_{\widehat{M}} u L_{\hat{g}} u d \widehat{V}=\lim _{j \rightarrow \infty} \int_{\widehat{M}} u_{j} L_{\hat{g}} u_{j} d \widehat{V} \\
& =\lim _{j \rightarrow \infty} \int_{\widehat{M}}\left(\left|\nabla u_{j}\right|_{\hat{g}}^{2}+\widehat{S} u_{j}^{2}\right) d \widehat{V}=\int_{\widehat{M}}\left(|\nabla u|_{\hat{g}}^{2}+\widehat{S} u^{2}\right) d \widehat{V},
\end{aligned}
$$

so $Q_{0}(\widehat{M}) \leq 0$. Thus $Q_{0}>0$ implies that $(1.5)$ for $\delta=0$ is an injection, and hence an isomorphism by Theorem 1.1. Using $W_{2, \delta}^{2} \subset W_{2,0}^{2}$ for $0<\delta<\nu_{0}$ and duality for $-\nu_{0}<\delta<0$, we obtain

Theorem 1.2. The operator (1.5) is an isomorphism for every nonnegative integer $s$ provided $Q_{0}(\widehat{M})>0, n \leq(N-2) / 2$, and $-\nu_{0}<\delta<\nu_{0}$.

\section{A SoboleV INEQUALITY AND REgULARITY AT INFINITY $(r=0)$}

In this section we obtain values of $s$ and $\delta$ that guarantee $u \in W_{s, \delta}^{2}(\widehat{M})$ is a continuous function vanishing at infinity. This was considered by Cantor [C] for the case $n=0$ and $\widehat{M}=\mathbb{R}^{N}$; our treatment parallels his in some respects. We shall first state a lemma holding in $U$ (i.e., near infinity of $\widehat{M}$ ) before 
stating the theorem on $\widehat{M}$. For the lemma we consider $u=u(\theta, \xi) \in C_{0}^{\infty}(U)$ and for fixed $\theta_{0} \in \Gamma$ we consider $u\left(\theta_{0}, *\right)$ on $N_{\theta_{0}}(\Gamma)$ and the norm

$$
\left\|u\left(\theta_{0}, *\right)\right\|_{s, \delta}^{2}=\sum_{l=0}^{s} \int_{|\xi|<\varepsilon}|\xi|^{-2 \delta}\left|\nabla_{\xi}^{l} u\left(\theta_{0}, \xi\right)\right|_{\hat{\xi}}^{2} d \widehat{V}_{\xi}
$$

where $d \widehat{V}_{\xi}$ is the measure induced by $\hat{g}$ on $N_{\theta_{0}}(\Gamma)$.

Lemma 2.1. Suppose $s>(N-n) / 2$ and $\beta<\delta+n / 2$. There is a constant $C_{0}>0$ such that for each $\theta_{0} \in \Gamma$

$$
\sup _{0<|\xi|<\varepsilon}\left|r^{-\beta} u\left(\theta_{0}, \xi\right)\right| \leq C_{0}\left\|u\left(\theta_{0}, *\right)\right\|_{s, \delta}
$$

for all $u \in C_{0}^{\infty}(U)$.

Proof. It suffices to prove $(2.1)$ for $(N-n) / 2<s \leq N-n$. Since $\Gamma$ is compact, it also suffices to prove (2.1) with $C_{0}=C_{0}\left(\theta_{0}\right)$. Thus we may restrict our attention to $\xi \in N_{\theta_{0}}(\Gamma)$, and for notational convenience in this proof we shall write $u(\xi)$ instead of $u\left(\theta_{0}, \xi\right)$. Moreover, the metric $g$ on $|\xi|<\varepsilon$ is equivalent (in terms of volume and length but not curvature) to the Euclidean metric, so for this proof we shall use $|\xi|$ to denote Euclidean length and $|\xi|_{g}$ to denote the length in the metric $g:|\xi| \approx|\xi|_{g}, d V \approx|\xi|^{N-n-1} d|\xi| d \omega d \theta$, etc.

For technical reasons, it is simpler to treat $\xi$ near infinity (cf. [C]) than near 0 , so we shall make the change of variables $y=\xi /|\xi|^{2}$ so that $u$ is supported near $y=\infty$. Fix $\xi_{0}$ with $0<\left|\xi_{0}\right|_{g}<\varepsilon$, let $y_{0}=\xi_{0} /\left|\xi_{0}\right|^{2}$, and choose $e \in N_{\theta_{0}}(\Gamma)$ with $|e|=1$ so that

$$
\left|\xi_{0}\right|^{-\beta} u\left(\xi_{0}\right)=\left|y_{0}\right|^{\beta} u\left(y_{0}\right)=\int_{0}^{\infty} \frac{d}{d t}\left[\left|y_{0}+t e\right|^{\beta} u\left(y_{0}+t e\right)\right] d t .
$$

Integrate by parts to get

$$
\begin{aligned}
\left|\xi_{0}\right|^{-\beta} u\left(\xi_{0}\right) & =\int_{0}^{\infty} C_{s} \frac{d^{s}}{d t^{s}}\left[\left|y_{0}+t e\right|^{\beta} u\left(y_{0}+t e\right)\right] t^{s-1} d t \\
& =\int_{0}^{\infty} \sum_{l=0}^{s} c_{l s} \frac{d^{l}}{d t^{l}} u\left(y_{0}+t e\right) \frac{d^{s-l}}{d t^{s-l}}\left|y_{0}+t e\right|^{\beta} t^{s-1} d t
\end{aligned}
$$

Thus

$$
\left|\xi_{0}\right|^{-\beta}\left|u\left(\xi_{0}\right)\right| \leq C_{1} \int_{0}^{\infty} \sum_{l=0}^{s}\left|\frac{d^{l}}{d t^{l}} u\left(y_{0}+t e\right)\right| \cdot\left|\frac{d^{s-l}}{d t^{s-l}}\right| y_{0}+\left.t e\right|^{\beta} \mid t^{s-1} d t .
$$

Now integrate both sides over $|e|=1$ to get

$$
\left|\xi_{0}\right|^{-\beta}\left|u\left(\xi_{0}\right)\right| \leq C_{2} \int_{|e|=1} \int_{0}^{\infty} \sum_{l=0}^{s}\left|\frac{d^{l}}{d t^{l}} u\left(y_{0}+t e\right)\right| \cdot\left|\frac{d^{s-l}}{d t^{s-l}}\right| y_{0}+\left.t e\right|^{\beta} \mid t^{s-1} d t d \omega .
$$

We may compute $|d u / d t|=\left|\nabla_{y} u \cdot e\right| \leq\left|\nabla_{y} u\right|_{g}|e|_{g} \approx|y|\left|\nabla_{y} u\right|_{\hat{g}}$ and $(d / d t) \mid y_{0}+$ $\left.t e\right|^{\alpha} \approx\left|y_{0}+t e\right|^{\alpha-1}$ to obtain

$$
\left|\xi_{0}\right|^{-\beta}\left|u\left(\xi_{0}\right)\right| \leq C_{3} \int_{|y|>1 / \varepsilon} \sum_{l=0}^{s}|y|^{l} \cdot\left|\nabla_{y}^{l} u(y)\right|_{\hat{g}} \cdot|y|^{\beta-s+l}\left|y-y_{0}\right|^{s+n-N} d y
$$


where $y=y_{0}+t e$ and $d y=t^{N-n-1} d t d \omega$ (Euclidean measure). Using the Schwarz Inequality,

$$
\begin{aligned}
& \int_{|y|>1 / \varepsilon}|y|^{2 l+\beta-s} \cdot\left|\nabla_{y}^{l} u(y)\right|_{\hat{g}} \cdot\left|y-y_{0}\right|^{s+n-N} d y \\
& \leq\left(\int_{|y|>1 / \varepsilon}|y|^{2 \delta+4 l} \cdot\left|\nabla_{y}^{l} u(y)\right|_{\hat{g}}^{2} \cdot|y|^{-N+2 n} d y\right)^{1 / 2} \\
& \cdot\left(\int_{|y|>1 / \varepsilon}|y|^{2(\beta-\delta-s-n)+N} \cdot\left|y-y_{0}\right|^{2(s+n-N)} d y\right)^{1 / 2} .
\end{aligned}
$$

Now $\left|\nabla_{y} u\right|_{\hat{g}} \approx|y|^{-2}\left|\nabla_{\xi} u\right|_{\hat{g}}$ and $|y|^{-N+2 n} d y \approx d \widehat{V}_{\xi}$ so

$$
\int_{|y|>1 / \varepsilon}|y|^{2 \delta+4 l} \cdot\left|\nabla_{y}^{l} u(y)\right|_{\hat{g}}^{2} \cdot|y|^{-N+2 n} d y \leq C \int_{|\xi|<\varepsilon}|\xi|^{-2 \delta} \cdot\left|\nabla_{\xi}^{l} u(\xi)\right|_{\hat{g}}^{2} d \widehat{V}_{\xi} .
$$

Combining this with (2.2) and (2.3) we see that we need only verify that

$$
\int_{|y|>1 / \varepsilon}|y|^{2(\beta-\delta-s-n)+N} \cdot\left|y-y_{0}\right|^{2(s+n-N)} d y
$$

is bounded independent of $\left|y_{0}\right|>1 / \varepsilon$. (This was claimed for $n=0$ by Cantor [C] without rigorous proof; in fact, his argument would suggest that (2.4) is independent of $y_{0}$ for every $s>(N-n) / 2$, but this is only true for $(N-n) / 2<$ $s \leq N-n$.)

But if $s=N-n$, then (2.4) is clearly independent of $y_{0}$. It is easy to check the one-dimensional estimate $\int_{1 / \varepsilon}^{\infty} y^{\alpha}\left|y-y_{0}\right|^{\gamma} d y \leq C$ for $-1<\gamma<0$ and $\alpha+\gamma<-1$. Then the $(N-n)$-dimensional case follows from $|y|^{N-n} \geq$ $\prod_{i=1}^{N-n}\left|y_{i}\right|$, where $y=\left(y_{1}, \ldots, y_{N-n}\right) \in \mathbb{R}^{N-n}$, and $|y|^{\alpha} \leq \prod_{i=1}^{N-n}\left|y_{i}\right|^{\alpha /(N-n)}$, $\left|y-y_{0}\right|^{\gamma} \leq \prod_{i=1}^{N-n}\left|y_{i}-y_{0 i}\right|^{\gamma /(N-n)}$, so we have $\int_{|y|>1 / \varepsilon}|y|^{\alpha}\left|y-y_{0}\right|^{\gamma} d y \leq C$ for $-(N-n)<\gamma<0$ and $\alpha+\gamma<-(N-n)$. Thus we obtain (2.4) with $\gamma=$ $2(s+n-N)$ and $\alpha=2(\beta-\delta-s-n)+N$ since $(N-n) / 2<s<N-n$ and $\beta<\delta+n / 2$. The lemma is proved. Now return to the notation $|\xi|^{2}=g_{i j} \xi^{i} \xi^{j}$.

Using Lemma 2.1 we will prove

Theorem 2.2. If $u \in W_{s, \delta}^{2}(\widehat{M})$, where $s>N / 2$ and $\delta \in \mathbb{R}$, then $u$ is a continuous function on $\widehat{M}$ and $r^{-\beta} u=o(1)$ as $r \rightarrow 0$ whenever $\beta<\delta+n / 2$.

Proof. We know $u \in C^{0}(\widehat{M})$ by the Sobolev Embedding Theorem, so we need only to show $r^{-\beta} u\left(\theta_{0}, \xi\right) \rightarrow 0$ as $|\xi| \rightarrow 0$ in $N_{\theta_{0}}(\Gamma)$ for each $\theta_{0} \in \Gamma$. As in the previous proof, we shall operator only on $N_{\theta_{0}}(\Gamma)$.

Pick $\chi \in C^{\infty}[0, \infty)$ with $\chi(r) \equiv 1$ for $0 \leq r<\varepsilon / 3$ and $\chi(r) \equiv 0$ for $r>2 \varepsilon / 3$. Define $\chi_{k}(\xi)=\chi(k \cdot|\xi|)$ so that $\chi_{k}(\xi)=0$ for $|\xi|>2 \varepsilon / 3 k$ and $\left|\nabla_{\xi}^{l} \chi_{k}\right| \leq C_{l} k^{l}$. But for $l>0, \nabla_{\xi}^{l} \chi_{k}$ is supported in $\varepsilon / 3 k<|\xi|<2 \varepsilon / 3 k$. Thus

$$
\left|\nabla_{\xi}^{l} \chi_{k}\right|_{\hat{g}}=|\xi|^{l}\left|\nabla_{\xi}^{l} \chi_{k}\right| \leq C_{l}^{\prime}
$$

holds for $l=0,1, \ldots, s$. 
Since $u \in W_{s, \delta}^{2}(\widehat{M}) \cap C^{0}(\widehat{M})$ and we may assume $u\left(\theta_{0}, \xi\right)$ vanishes for $|\xi|>\varepsilon$, we have $\left\|u\left(\theta_{0}, *\right)\right\|_{s, \delta}$ finite. Moreover

$$
\begin{aligned}
\left\|\chi_{k}(*) u\left(\theta_{0}, *\right)\right\|_{s, \delta}^{2} & =\sum_{l=0}^{s}\left\|\nabla_{\xi}^{l}\left(\chi_{k}(*) u\left(\theta_{0}, *\right)\right)\right\|_{\delta}^{2} \\
& \leq \sum_{l=0}^{s} \sum_{\alpha=0}^{l}\left\|\nabla_{\xi}^{l-\alpha} \chi_{k}(*) \nabla_{\xi}^{\alpha} u\left(\theta_{0}, *\right)\right\|_{\delta}^{2} .
\end{aligned}
$$

But using (2.5),

$$
\left\|\nabla_{\xi}^{l-\alpha} \chi_{k}(*) \nabla_{\xi}^{\alpha} u\left(\theta_{0}, *\right)\right\|_{\delta}^{2} \leq C_{l-\alpha}^{\prime} \int_{|\xi|<\varepsilon / k}|\xi|^{-2 \delta}\left|\nabla_{\xi}^{\alpha} u\left(\theta_{0}, \xi\right)\right|_{\hat{g}}^{2} d \widehat{V}_{\xi} \rightarrow 0 \text { as } k \rightarrow \infty
$$

So we may use Lemma 2.1 to conclude that $r^{-\beta} u\left(\theta_{0}, \xi\right) \rightarrow 0$ as $|\xi| \rightarrow 0$. The theorem is proved.

\section{Proof of the MAIN THeorem}

Recall that $(\widehat{M}, \hat{g})$ is a complete Riemannian manifold with a warped cylindrical end and $\hat{g}$ is conformal to $g$ on $\widehat{M}$. If we can solve $L_{\hat{g}} v=0$ with $v \geq \varepsilon>0$ on $\widehat{M}$, then the metric $\tilde{g}=v^{4 /(N-2)} \hat{g}$ will be complete, conformal to $g$, and have zero scalar curvature, so we will have proved our theorem.

Write $v=v_{0}+v_{1}$ where $v_{0}$ is a smooth positive function satisfying $v_{0}=$ $r^{-\nu_{0}+n / 2}$ near $r=0$ : since $n \leq(N-2) / 2$ implies $\widehat{S}_{0} \geq 0$ and hence $\nu_{0} \geq n / 2$ (cf. (1.3)), we see that $v_{0} \geq \varepsilon>0$ on $\widehat{M}$.

Next we must compute $L_{\hat{g}} v_{0}$. Recall that $\hat{g}$ is asymptotic to $\hat{g}_{0}=r^{-2} d r^{2}+$ $r^{-2} d \theta^{2}+d \omega^{2}$ as $r \rightarrow 0$. More precisely, if we write $d \theta^{2}=g_{\alpha \beta} d \theta^{\alpha} d \theta^{\beta}$ and $d \omega^{2}=g_{\mu \nu} d \omega^{\mu} d \omega^{\nu}$ (where $1 \leq \alpha, \beta \leq n$ and $n+1 \leq \mu, \nu \leq N-1$ ), then

$$
\begin{aligned}
\hat{g}= & \left(r^{-2} g_{\alpha \beta}(\theta)+O\left(r^{-1}\right)\right) d \theta^{\alpha} d \theta^{\beta}+\left(g_{\mu \nu}(\omega)+O\left(r^{2}\right)\right) d \omega^{\mu} d \omega^{\nu}+r^{-2} d r^{2} \\
& +O\left(r^{-1}\right) d r d \theta^{\alpha}+O(1) d \theta^{\alpha} d \omega^{\mu}
\end{aligned}
$$

where $O\left(r^{q}\right)$ means terms for which all derivatives $r \partial_{r}, \partial_{\omega}$, and $r \partial_{\theta}$ are also $O\left(r^{q}\right)$ as $r \rightarrow 0$ (see [MM, §7] for more details). We may then compute

$$
\operatorname{det} \hat{g}=r^{-2 n-2}\left(\operatorname{det}\left(g_{\alpha \beta}\right)\right)\left(\operatorname{det}\left(g_{\mu \nu}\right)\right)+O\left(r^{-2 n-1}\right)
$$

so that

$$
\begin{aligned}
\sqrt{\operatorname{det} \hat{g}} & =r^{-n-1}\left[\left(\operatorname{det}\left(g_{\alpha \beta}\right)\right)\left(\operatorname{det}\left(g_{\mu \nu}\right)\right)+O\left(r^{-2 n-1}\right)\right]^{1 / 2} \\
& =r^{-n-1}\left(\operatorname{det}\left(g_{\alpha \beta}\right)\right)^{1 / 2}\left(\operatorname{det}\left(g_{\mu \nu}\right)\right)^{1 / 2}+O\left(r^{-n}\right)
\end{aligned}
$$

and

$$
1 / \sqrt{\operatorname{det} \hat{g}}=r^{n+1}\left(\operatorname{det}\left(g_{\alpha \beta}\right)\right)^{-1 / 2}\left(\operatorname{det}\left(g_{\mu \nu}\right)\right)^{-1 / 2}+O\left(r^{n+2}\right) .
$$

If we use matrix notation,

$$
\left(\hat{g}_{i j}\right)_{i, j=1}^{N}=\left[\begin{array}{ccc}
r^{-2} g_{\alpha \beta}+O\left(r^{-1}\right) & O(1) & O\left(r^{-1}\right) \\
O(1) & g_{\mu \nu}+O\left(r^{2}\right) & 0 \\
O\left(r^{-1}\right) & 0 & r^{-2}
\end{array}\right]
$$


we may compute the inverse matrix

$$
\left(\hat{g}^{i j}\right)_{i, j=1}^{N}=\left[\begin{array}{ccc}
r^{2} g^{\alpha \beta}+O\left(r^{3}\right) & O\left(r^{2}\right) & O\left(r^{3}\right) \\
O\left(r^{2}\right) & g^{\mu \nu}+O(r) & O\left(r^{3}\right) \\
O\left(r^{3}\right) & O\left(r^{2}\right) & r^{2}+O\left(r^{4}\right)
\end{array}\right]
$$

A calculation shows that we may write

$$
\Delta_{\hat{g}}=\Delta_{\hat{g}_{0}}+O(r) P_{2}\left(r \partial_{r}, r \partial_{\theta}, \partial_{\omega}\right)
$$

where $P_{2}$ is a quadratic polynomial in its variables. Using (1.2) we have $\widehat{S}=$ $\widehat{S}_{0}+O\left(r^{2}\right)$ so that

$$
f=-L_{\hat{g}} v_{0}=\left(\Delta_{\hat{g}_{0}}-\widehat{S}_{0}\right) v_{0}+O(r) v_{0}=O\left(r^{-\nu_{0}+n / 2+1}\right) .
$$

Notice that $f \in W_{s, \delta}^{2}(\widehat{M})$ for every $s$ provided $\delta<1-\nu_{0}$. Using Theorem 1.2 we may solve $L_{\hat{g}} v_{1}=f$ with $v_{1} \in W_{s+2, \delta}^{2}(\widehat{M})$ for every $s$, provided

$$
-\nu_{0}<\delta<\min \left(\nu_{0}, 1-\nu_{0}\right) \text {. }
$$

But $-\nu_{0}<\delta$ together with Theorem 2.2 imply that $r^{\nu_{0}-n / 2} v_{1} \rightarrow 0$ as $r \rightarrow 0$, so $v=v_{0}+v_{1} \approx r^{-\nu_{0}+n / 2}$ as $r \rightarrow 0$. In particular, $v$ is positive near infinity of $\widehat{M}$, but $Q_{0}(\widehat{M})>0$ implies $\lambda_{0}\left(M_{0}, \hat{g}\right)>0$, where $M_{0}$ is compact in $\widehat{M}$, and the Generalized Maximum Principle of [PW] shows that $v>0$ on $M_{0}$, so $v \geq \varepsilon>0$ on $\widehat{M}$ as desired.

\section{REFERENCES}

[AM] P. Aviles and R. C. McOwen, Complete conformal metrics with negative scalar curvature in compact Riemannian manifolds, Duke Math. J. 56 (1988), 395-398.

[C] M. Cantor, Elliptic operators and the decomposition of tensor fields, Bull. Amer. Math. Soc. 5 (1981), 235-262.

[GT] D. Gilbarg and N. Trudinger, Elliptic partial differential equations of second order, 2nd edition, Springer-Verlag, Berlin, Heidelberg, New York, and Tokyo, 1983.

[J] Zhiren Jin, A counterexample to the Yamabe problem for complete noncompact manifolds, Lecture Notes in Math., vol. 1306, Springer-Verlag, Berlin, Heidelberg, New York, London, Paris, and Tokyo, 1988, pp. 93-101.

[LN] C. Loewner and L. Nirenberg, Partial differential equations invariant under conformal or projective transformations, Contributions to Analysis, Academic Press, New York, 1974, pp. 245-272.

[LP] J. M. Lee and T. H. Parker, The Yamabe problem, Bull. Amer. Math. Soc. 17 (1987), 37-91.

[MM] X. Ma and R. C. McOwen, The Laplacian on complete manifolds with warped cylindrical ends, Commun. in Partial Differential Equations, 16(10), 1583-1614 (1991).

[PW] M. Protter and H. Weinberger, Maximum principles in differential equations, Prentice-Hall, Englewood Cliffs, NJ, 1967.

Department of Mathematics, University of Wisconsin, La Crosse, Wisconsin 54601

Department of Mathematics, Northeastern University, Boston, MasSachusetTs 02115 E-mail address: McOWEN@NUHUB.BITNET 